\title{
A commentary on: "A pan-cancer single-cell transcriptional atlas of tumor infiltrating myeloid cells" - tumor microenvironment: the Achilles heel of cancer
}

https://doi.org/10.1515/mr-2021-0032

Received November 19, 2021; accepted December 13, 2021;

published online February 22, 2022

\section{Significance of tumor microenvironment (TME) to cancer}

Cancer is a complicated disease. With the deepening of the research on the mechanism of tumor initiation, promotion and progression in recent years, researchers have gradually shifted their focus from tumor itself to TME. The concept of TME can be traced back to the relationship between tumor and inflammation and the theory of "seed and soil" in the latter half of the nineteenth century [1]. TME consists of non-malignant cells, vessels, lymph nodes, nerves, intercellular components, and metabolites located at the center, margin, or periphery of the tumor [1]. There is increasing evidence that cellular and non-cellular components of the TME can promote tumor initiation, growth, invasion, metastasis, and response to treatment. The TME is composed of three main cell types: immune cells, stromal cells (e.g. cancer-associated fibroblasts), vascular cells [2, 3]. For the past few years, the interaction between tumor cells and immune cells has been gradually recognized. Tumors are known to change markedly in terms of the quantity and phenotypes of immune and stromal cells

\footnotetext{
*Corresponding authors: Zhaode Bu and Jiafu Ji, Gastrointestinal Cancer Center, Key Laboratory of Carcinogenesis and Translational Research (Ministry of Education), Peking University Cancer Hospital \& Institute, Beijing 100142, China, E-mail: buzhaode@cjcrcn.org (Z. Bu), jijiafu@hsc.pku.edu.cn (J. Ji)

Jingtao Wei, Gastrointestinal Cancer Center, Key Laboratory of Carcinogenesis and Translational Research (Ministry of Education), Peking University Cancer Hospital \& Institute, Beijing, China. https:// orcid.org/0000-0001-8260-1835
}

in the TME [3]. TME is highly heterogeneous across different patients, and the plasticity of immune cells is an important part of tumor heterogeneity. The immunosuppression after immune evasion becomes a new hallmark of cancer [2]. The infiltration of immune cells in TME, located at the core or margin of tumor, the adjacent lymphoid organ or lymph nodes (also called tertiary lymphoid structures), is closely related to the prognosis of cancer [1].

Immune cells in TME can be divided into lymphocytes and myeloid cells according to their diverse developmental lineages. Immunosuppressive myeloid cells are one of the major obstacles to cancer immunotherapy. At present, although several therapeutic approaches targeting myeloid cells are being tested in preclinical and clinical studies, the study of lymphocytes is relatively in-depth [4]. Tumor-infiltrating lymphocytes consist of all lymphocytic cell populations that have invaded the tumor tissue, including T cells, B cells, plasma cells and natural killer (NK) cells. T cell-mediated adaptive immunity is considered to play a major role in anti-tumor immunity [5]. According to function, immune cells can be classified as tumor-antagonizing and tumor-promoting immune cells. The tumor-antagonizing immune cells mainly consist of effector $\mathrm{T}$ cells (including $\mathrm{CD}^{+}$cytotoxic $\mathrm{T}$ cells and effector $\mathrm{CD}^{+} \mathrm{T}$ cells), NK cells, dendritic cells (DCs), M1-like macrophages and N1-like neutrophils [2]. The CD8 ${ }^{+}$ cytotoxic $\mathrm{T}$ cells are considered to be the major subset of lymphocytes that kill cancer cells. NK cells are also one important subset of tumor-antagonizing immune cells that mediate the immunosurveillance of tumor [2]. On the other hand, $\mathrm{T}$ regulatory cells (Tregs) are able to inhibit the immune response mediated by $\mathrm{CD}^{+}$and $\mathrm{CD}^{+} \mathrm{T}$ cells, by suppressing $\mathrm{T}$ cell proliferation, antigen presentation, and cytokine production [6]. The recent single-cell sequencing revealed that $\mathrm{T}$ cells are highly heterogeneous and $\mathrm{T}$ cell states associated with the prognosis of patients and therapy response [7]. In a part of tumors, the tumor cells would inhibit the activation of cytotoxic $\mathrm{T}$ cells via expressing 
inhibitory ligands such as PD-L1 or CTLA-4, which allows tumor cells to escape from host immune surveillance and resistant to cancer therapy [2]. Furthermore, immunotherapy targeting $\mathrm{T}$ cells has made great progress and gradually become a new therapy for various tumors. And the research targeting myeloid cells could also promote current immunotherapies.

\section{Tumor-infiltrating myeloid cells (TIMs) are an important component of TME}

Myeloid cells constitute another type of important immune cells in the TME and also work as key players in the innate immune system. The tumor could recruit and modulate endogenous myeloid cells, including macrophages, DCs, myeloid-derived suppressor cells (MDSC) and neutrophils, to sustain an immunosuppressive environment [8]. However, compared with lymphocytes, the heterogeneity of myeloid cells is relatively poorly studied, and there is also a lack of widely effective drugs targeting TIMs. Encouragingly, a recent study [9] revealed the heterogeneity of myeloid cells across different tumors from the perspective of single cell.

It is commonly believed that tumor-associated macrophages (TAMs) could promote chronic inflammation, inhibit the anti-tumor immune response of $\mathrm{T}$ cells, support tumor growth through angiogenesis and promote metastasis [8]. While TAM infiltration usually correlates with a poor outcome, correlations between high TAM density and improved survival are also emerged [10]. In the past, the classical ("M1") or alternative ("M2") activation has been used to describe the in vitro state of macrophages. However, it is now recognized that macrophages exhibit more complex functional phenotypes in vivo. The study found that pro-angiogenic TAMs exist in different types of cancer, which are associated with poor prognosis, but they present different markers [9]. Therefore, immunotherapy targeting those angiogenesis-associated macrophages should take their diverse characteristics in various cancer types into consideration.

Similarly, conventional DCs exhibit diversity and could be divided into $\mathrm{CDC} 1$ and $\mathrm{CDC} 2$ with different functional specialization. cDC1s are mainly responsible for antigen cross-presentation and are critical for initiating $\mathrm{CD}^{+} \mathrm{T}$ cell response [10]. While, cDC2s mainly activate $\mathrm{CD}^{+} \mathrm{T}$ cells through presentation of peptides on
MHC-II [10]. Besides, the complexity of cDC2s across tumors is still not fully characterized. By single cells RNA sequencing (scRNA-seq), three distinct subsets in cDCs were identified, including two classical $\mathrm{cDC}$ subsets (cDC1s and $\mathrm{cDC} 2 \mathrm{~s})$ and a mature $\mathrm{CDC}$ subset $\left(L A M P 3^{+}\right.$ $\mathrm{CDC}$ ), which is a new subset previously reported in hepatocellular carcinoma $[9,11]$. Moreover, the study proved that the $L A M P 3^{+}$cDCs were broadly present and CDC1derived and $\mathrm{CDC} 2$-derived $L A M P 3^{+} \mathrm{CDCs}$ are regulated by different external stimulus and might have diverse functions [9].

Although mast cells are one important component of TIMs, they are still poorly studied compared to others [12]. Within the TME, mast cells could possess both tumorantagonizing and tumor-promoting properties through different approaches, including angiogenesis, tissue remodeling, disruption of the extracellular matrix, invasion, and metastatic spread [12]. This study also made a great breakthrough in exploring the functional diversity of mast cells in TME. The frequencies of $V E G F A^{+}$mast cells were much higher than $T N F^{+}$cells in most tumor types, indicating their prevailing pro-angiogenic role. On the contrary, nasopharyngeal cancer was the only cancer type with dramatically higher frequency of $T N F^{+}$mast cells, indicating a higher anti-tumor capacity [9].

However, neutrophils, an important component of myeloid cells, were not studied in detail. Compared with other immune cells, the number and level of gene expression in neutrophils are lower and mRNA capture is more difficult. On the other hand, neutrophils are sensitive to degradation and have a short half-life, which requires high requirements for experimental operation. So scRNA-seq of neutrophils requires specific optimization steps.

\section{Immunotherapy targeting TME}

Nowadays, the goal of cancer immunotherapy is to turn cold tumors into hot ones and break the immunosuppressive TME. Immunotherapy such as checkpoint inhibitors targeting $\mathrm{T}$ lymphocytes has been widely used in clinical practice. But immunotherapy targeting myeloid cells is still in its infancy $[4,8,10]$. The pan-cancer singlecell transcriptional atlas of TIMs provides the basis for subsequent translational research, exploration of therapeutic targets and selection of adaptation population.

There are several strategies for breaking immunosuppression in TME: controlling cancer-promoting inflammation; blocking the recruitment or survival of myeloid cells; 
altering the activation status of pro-tumorigenic myeloid cells and reprogramming of a pro-tumorigenic phenotype of myeloid cells $[4,10]$. On the other hand, many of these targets and clinical trials did not target specific subpopulations of myeloid cells and may cause some side effects and toxicity. This study provided a better understanding of the functional diversity and heterogeneity of myeloid subpopulation across different types of cancer and laid the foundation for the identification of novel targets specifically expressed on unique myeloid subsets, such as macrophages, mast cells, and dendritic cells.

Research funding: None declared.

Author contributions: All authors have accepted responsibility for the entire content of this manuscript and approved its submission.

Competing interests: Authors state no conflict of interest. Informed consent: Not applicable.

Ethical approval: Not applicable.

\section{References}

1. Jin MZ, Jin WL. The updated landscape of tumor microenvironment and drug repurposing. Signal Transduct Target Ther 2020;5:166.
2. Lei X, Lei Y, Li JK, Du WX, Li RG, Yang J, et al. Immune cells within the tumor microenvironment: biological functions and roles in cancer immunotherapy. Cancer Lett 2020;470:126-33.

3. Schmitt M, Greten FR. The inflammatory pathogenesis of colorectal cancer. Nat Rev Immunol 2021;21:653-67.

4. Nakamura K, Smyth MJ. Myeloid immunosuppression and immune checkpoints in the tumor microenvironment. Cell Mol Immunol 2020;17:1-12.

5. Rojas A, Araya P, Gonzalez I, Morales E. Gastric tumor microenvironment. Adv Exp Med Biol 2020;1226:23-35.

6. Oya Y, Hayakawa Y, Koike K. Tumor microenvironment in gastric cancers. Cancer Sci 2020;111:2696-707.

7. van der Leun AM, Thommen DS, Schumacher TN. CD8(+) T cell states in human cancer: insights from single-cell analysis. Nat Rev Cancer 2020;20:218-32.

8. Schupp J, Krebs FK, Zimmer N, Trzeciak E, Schuppan D, Tuettenberg A. Targeting myeloid cells in the tumor sustaining microenvironment. Cell Immunol 2019;343:103713.

9. Cheng S, Li Z, Gao R, Xing B, Gao Y, Yang Y, et al. A pan-cancer single-cell transcriptional atlas of tumor infiltrating myeloid cells. Cell 2021;184:792-809 e23.

10. Jahchan NS, Mujal AM, Pollack JL, Binnewies M, Sriram V, Reyno L, et al. Tuning the tumor myeloid microenvironment to fight cancer. Front Immunol 2019;10:1611.

11. Zhang Q, He Y, Luo N, Patel SJ, Han Y, Gao R, et al. Landscape and dynamics of single immune cells in hepatocellular carcinoma. Cell 2019;179:829-45 e20.

12. Aponte-Lopez A, Munoz-Cruz S. Mast cells in the tumor microenvironment. Adv Exp Med Biol 2020;1273:159-73. 